\title{
Functional capacity and dual-task cost in the institutionalized older adults, both affected and unaffected by mild cognitive impairment
}

\author{
Marek Zak ${ }^{1 *}$ D, Szymon Krupnik², Waldemar Brola', Dorota Rebak', Tomasz Sikorski ${ }^{1}$, Frederic Dutheil ${ }^{3,4}$,
} Jaroslaw Andrychowski and Daniel Courteix ${ }^{5}$

\begin{abstract}
Background: Mild cognitive impairment (MCl) affects 10-20\% of the individuals over the age of 65 ; this proportion being higher in the institutional care facilities than within a general population.

Aim: To assess whether dual-task cost in the individuals affected by $\mathrm{MCl}$ depends exclusively on gait, or possibly some other functional capacity components might also come into play, as compared to the healthy controls also remaining in the institutional care.

Methods: The study was conducted in five nursing facilities, involving 88 subjects in total, i.e. 44 subjects affected by $\mathrm{MCl}$ (mean age of 83.8 years; 34 women (77.3\%) and 10 men (22.7\%), and 44 healthy controls (mean age 81.67 years; 38 women (84.4\%) and 7 men (15.6\%). Cognitive functions were assessed through Mini-Mental State Examination (MMSE), while gait by Timed Up and Go Test (TUGT). Gait speed was calculated by the 10 Meter Walk Test, and the fear of falling with the Falls Efficacy Scale International. Dual tasks were assessed by TUGT MAN (Timed Up and Go Test Manual) and TUGCog (Timed Up and Go Test Cognitive). Dual Task Cost (DTC) of TUGT MAN and TUGT $_{\text {COG }}$ was established. Statistical analyses were completed with STATISTICA Package v. 10.

Results: Individuals affected by $\mathrm{MCl}$ differed significantly from the unaffected ones with regard to their gait test results, when assigned a single-task activity, and dual-task activities, as well as in the gait speed. Dual Task Cost Manual (DTC MAN) in the $\mathrm{MCl}$ group was significantly higher, as compared to the subjects unaffected by MCl. Around $25 \%$ of the variance of DTC MAN result regarding the $\mathrm{MCl}$ group was accounted for by gait performance in the single-task conditions (TUGT). In the case of Dual Task Cost Cognitive (DTC $\mathrm{COG}_{\text {) }}$, this value equalled to approx. 10\%. A 1\% change in DTC MAN corresponded to approx. $0.5 \mathrm{~s}$ change in TUGT, whereas a 1\% change in DTC COG entailed approx. $0.35 \mathrm{~s}$ change in TUGT walking time.
\end{abstract}

Conclusion: Individual functional capacity affected the dual-task performance, especially the motor-motor tasks. Dual-task cost in the subjects affected by $\mathrm{MCl}$ was significantly reduced, being more dependent on the gait speed in the motor-motor tasks, which entailed visual memory, than in the motor-cognitive tasks.

Keywords: Older adults, MCl, Dual-task, Institutionalised care, Public health

\footnotetext{
* Correspondence: mzak1@onet.eu

'The Institute of Health Sciences, Collegium Medicum, The Jan Kochanowski University, ul. Zeromskiego 5, 25-369 Kielce, Poland

Full list of author information is available at the end of the article
}

C C The Author(s). 2021 Open Access This article is licensed under a Creative Commons Attribution 4.0 International License, which permits use, sharing, adaptation, distribution and reproduction in any medium or format, as long as you give appropriate credit to the original author(s) and the source, provide a link to the Creative Commons licence, and indicate if changes were made. The images or other third party material in this article are included in the article's Creative Commons licence, unless indicated otherwise in a credit line to the material. If material is not included in the article's Creative Commons licence and your intended use is not permitted by statutory regulation or exceeds the permitted use, you will need to obtain permission directly from the copyright holder. To view a copy of this licence, visit http://creativecommons.org/licenses/by/4.0/. The Creative Commons Public Domain Dedication waiver (http://creativecommons.org/publicdomain/zero/1.0/) applies to the data made available in this article, unless otherwise stated in a credit line to the data. 


\section{Introduction}

Mild cognitive impairment (MCI) is defined as cognitive impairment related to individual age- and educationrelated abilities, which has no bearing on executing the activities of daily living (ADLs) [1]. MCI affects 10-20\% of the individuals over the age of $65[2,3]$. Cahill et al. demonstrated that approx. $89 \%$ of institutionalized persons experienced mild to severe symptoms of dementia [4]. There is a correlation between cognitive function and gait, since - as demonstrated by Srygley et al. - gait is not a fully automatic activity. In fact, it also entails a cognitive component [5]. Montero-Odasso et al. demonstrated that individuals affected by MCI exhibited reduced stride time variability, while dual tasking [6]. In their study, poor executive functions, as well as working memory were associated with low gait speed.

Lee et al. demonstrated that Dual-task cost (DTC) in the individuals affected by MCI was greater with regard to regular gait, compared to those unaffected by this impairment, priority gait (two-fold greater), and cognitive prioritisation gait (three-fold greater) [7]. Also, the individuals exposed to Motoric Cognitive Risk (MCR) syndrome, construed as subjective cognitive complaints, a predictor of dementia, are characterised by reduced gait speed, information processing speed, and executive functions, without delayed free recall memory [8] when assigned a dual-task regimen [9]. Regardless of these reports, it has not been established beyond a reasonable doubt whether DTC in the individuals affected by MCI depends solely on gait, or whether other functional capacity components might also be entailed.

\section{Purpose}

The present study aimed therefore to establish conclusively whether DTC in the individuals affected by MCI depends exclusively on gait, or possibly some other functional capacity components might also come into play, thus making this relationship far more complex in terms of causal factors, as compared to the healthy controls unaffected by MCI. It was assumed that individuals affected by MCI had higher DTC in Timed Up and Go Test Manual (TUGT $T_{\text {MAN }}$ ) vs. Timed Up and Go Test Cognitive (TUGT $\mathrm{COG}_{\mathrm{C}}$ )

\section{Methods}

\section{Patients and methods}

The study was conducted in five nursing facilities, involving 88 subjects in total, i.e. 44 subjects with $\mathrm{MCI}$ (mean age of 83.8 years; 34 women $(77.3 \%$ ) and 10 men $(22.7 \%)$ ) and 44 healthy controls unaffected by $\mathrm{MCI}$ (mean age 81.67 years; 38 women $(84.4 \%)$ and 7 men (15.6\%)).

The following inclusion criteria were applied: age (> 65 years), absence of neurological or auditory deficits (i.e. a history of stroke with visible functional deficits of the locomotor system, sciatica, cerebral injuries), regular use of glasses with a power not exceeding 4 dioptres, and a Mini-Mental State Examination (MMSE) score of less than 24 points, although not less than 18, Barthel scale $<40$ points [10]. These criteria were supplemented by those proposed in the study by Winblad et al. [2] Furthermore, any elderly individuals who were found unable to complete any of the functional ability tests, were at that point pronounced non-eligible for further attendance in the study protocol.

Representativeness was calculated using a sample-size calculator:

(http://www.nss.gov.au/nss/home.nsf/pages/Sample+ size+calculator).

For a population of 500,000 (a proportion of 0.5 , and a standard error of 5.1), in conjunction with the 1.5 odds ratio $(\mathrm{OR})$ for a decrease in the gait speed in the group affected by $\mathrm{MCI}$, the determined representative population sample size was 44 subjects (MCI group), and 44 subjects unaffected by MCI (healthy controls group). The subjects were recruited by the onsite specialists employed in each nursing facility. Sociodemographic data of the individuals affected by MCI were acquired from their medical records. In the case of healthy controls, the data were acquired directly from the subjects. The variables addressing individual functional ability were presented as the data characterising both the study and the control groups. The testing protocol was executed by a trained physiotherapist boasting a minimum 2-year hands-on working experience with the elderly patients, and prior experience in pursuing academic research projects.

Prior to the actual commencement of the study proto$\mathrm{col}$, the subjects were verbally introduced to its practical specifics. A general presentation was made, whereas the key points of the testing procedure were shown in the form of pictograms. All applicable constraints for the execution of the tests were fully compliant with the breakdown provided further below.

At the First Session, pertinent sociodemographic data were collected, followed by the assessment of individual cognitive abilities against the MMSE. The score acquired in the MMSE test was a qualifier for further attendance in the study protocol. In practical terms, if an individual failed to score within the 18-23 points range, he was pronounced non-eligible for further participation in the study protocol.

Subsequently, Timed Up and Go Test (TUGT),

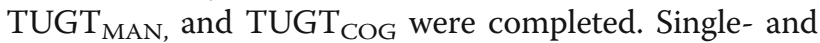
dual-task tests were carried out 3 times, whereas the mean score was ultimately recorded.

The Second Session comprised the 10 Meter Walk Test (10MWT) tests, both in the regular, and the fastest 
version, Strength of Lower Limbs and Fear of Falling (FOF), were also assessed.

The Third Session comprised the execution of 3 Single Leg Stance Open Eyes (SLS OP) and Single Leg Stance Closed Eyes (SLS CL) tests.

The examination of participants remaining in institutional care lasted $1.5 \mathrm{~h}$ in total, $30 \mathrm{~min}$ per each session.

Healthy subjects were tested in their own place of residence, in full compliance with the procedure outlined further above.

All participants were granted a $3-5 \mathrm{~min}$ period of rest between respective tests assessing individual functional performance. Subjects were assessed $2 \mathrm{~h}$ after a meal, in the morning hours.

\section{Cognitive functions}

Cognitive functions were assessed through MMSE, also known as the Folstein test, which facilitates assessment of five cognitive functions, i.e. orientation, memory, concentration, language, and constructional praxis [11].

The corresponding criterion for inclusion into the study group was the score ranging $18-23$ points, as per applicable guidelines in the literature on the subject, indicative of mild cognitive impairment [11]. The test was completed by a trained physiotherapist, under the same conditions for each subject.

Gait performance assessment under the single- and dualtask conditions

The version of TUGT test proposed by Podsiadlo was used to evaluate gait performance under the single-task conditions [12]. The subjects were advised that the correct way to perform the test was to stand up from a chair and then walk a 3-m distance behind the line, then turn around, walk all the way back, and sit down in the chair. The walk should be completed at the fastest possible pace deemed safe by the test subject. Under the dual-task conditions, gait performance was assessed by means of yet another version of the TUGT test, i.e. the one modified in line with Shumway-Cook and Wollacotte [13]. Dual-task activities were divided into the two types, i.e. motor-motor tasks (manual TUGT - TUGTMAN) and motor-cognitive tasks (cognitive TUGT TUGT $_{\mathrm{COG}}$ ). Both types of activities were performed following verbal and non-verbal instructions given by a physiotherapist.

During the TUGT $\mathrm{T}_{\text {MAN }}$ test, the subjects were asked to perform the same task as the TUGT test, while holding a beaker filled up with water in their dominant hand, whereas with regard to the TUGT $\mathrm{COG}_{\mathrm{CO}}$ test, the subjects were supposed to incrementally count down by 7 , starting off with a randomly selected number between 20 and 100, while walking. During either type of the dualtask test (TUGT $\mathrm{T}_{\text {MAN }}$ and TUGT $\mathrm{T}_{\mathrm{COG}}$ ), a physiotherapist did not indicate which task had a higher priority. All timings were taken with the aid of a mobile phone (Apple iPhone 6 - model A1586), and a built-in stopwatch application with an accuracy of $0.01 \mathrm{~s}$. A drop in the performance of dual-task activities was considered clinically significant, when the DTC, expressed as the relative difference between the gait speed under the dual-task conditions and that under the single-task ones, and then calculated in line with the formula given by Bock, was higher than 15\% [14]. In conformity with the algorithm proposed by Bock, an average DTC for healthy elderly individuals amounted to $15 \%$, having effectively been calculated out of 13 different dual-task activities; the same criterion was consequently assumed in this study protocol [14].

The gait speed corresponding to either the single-task, or the dual-task conditions was determined by dividing the distance covered during the TUGT, TUGT MAN $_{\text {and }}$ TUGT $_{\text {COG }}$ tests by the respective time it took to do so.

These tests were selected in view of being comprised of four essential components directly aiding the research effort, as well as offering the possibility of assessing a scope of ADLs:

1. Exclusion of the learning effect (TUGT test is performed first).

2. Very high test reproducibility [15].

3. An opportunity to assess short-term memory and metastability of attention during complex activity.

4. This test assesses the ability to stand up, walk, turn around, and sit down, i.e. an essential component in an individual pursuit of the ADLs.

\section{Gait speed}

Gait speed was calculated with the aid of the 10MWT, whereby the subject had to walk a distance of $10 \mathrm{~m}$. In line with the test methodology, the first and the final metre were excluded from the measurements [16]. Additionally, the two gait speeds were measured, i.e. normal, everyday speed, and the fastest speed the subject felt comfortable with.

\section{FOF}

The fear of falling was assessed by means of the Falls Efficacy Scale International Version (FES-I) $[17,18]$. This scale consists of 16 components, each of which is scored $1-4$ points, whereby a minimum score of 16 points indicates no fear of falling, whereas a maximum score of 64 points indicates high fear of falling $[17,18]$.

\section{Strength of the lower limbs}

The strength of the lower limbs was assessed using the 30-s chair stand test $(30 \mathrm{sChS})$, pursued in the way described by Jones and Rikli [19]. A physiotherapist counted aloud the number of repetitions, yet never 
verbally encouraged the subject to do any additional repetitions.

\section{Aerobic endurance}

Aerobic endurance was assessed with the aid of a 2Minute Step (2-Min. Step) test. In line with the methods proposed by Rikli and Jones [20], the subjects pursued a stationary walk for two minutes, while lifting their knees halfway between the patella and the iliac crest in a standing position. The outcome of the test was determined by the number of right limb raises above the line drawn on the wall, for each subject respectively.

\section{Static balance}

The single leg stance (SLS - Single Leg Stance) test was performed to assess static balance. The test was carried out under two different conditions, i.e. with the eyes open SLS OP, and then with the eyes closed SLS CL. The subject was to maintain balance while standing on the dominant leg [21]. The test ends when the foot touches the ground and the result is the actual duration (in seconds) of maintaining balance by the test subject.

\section{Statistical analysis}

Statistical data were processed with the aid of the STAT ISTICA 10 software package for Windows. Descriptive statistics were also completed. Normal/Gaussian distributions were calculated by means of the Shapiro-Wilk test $(n<100)$. Spearman's rank correlation coefficient was calculated to determine the level of interdependence of variables. The Mann-Whitney $U$ test was pursued for quantitative variables. DTC $(\%)=100 *$ (single task score dual task score)/single-task score [14].

An adjusted multi-nominal regression model was developed, with a view to indicating the effect of the independent variable TUGT on the dependent variable Dual Task Cost Manual (DTC $\mathrm{CAN}_{\mathrm{N}}$ ) in the subjects affected by MCI. The adjusted model was standardised against the select anthropometric data (i.e. sex, age, height, weight, and body mass index). The alpha significance level was set at $=0.05$.

\section{Results}

\section{Intergroup differences in the DTC}

Statistical analysis indicated that MCI subjects had a higher motor - motor cost (mean difference between the two groups 8.73 of DTC, the percentage differences between the two groups $104.3 \%, p<0.01$ ) and a lower motor-cognitive cost (mean difference between the two groups 13.2 of DTC, the percentage differences between the two groups $34.11 \%, p<0.05$ ), as compared to the control group. Significantly fewer subjects affected by MCI had a dual-task, motor-motor and cognitive-motor cost, as compared to the control group, by 63.5 and
$14.4 \%$, respectively. The MCI subjects differed in gait

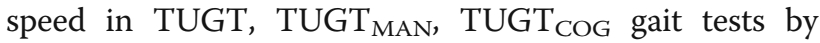
58.8, 86.7, and $57.7 \%$.

Baseline characteristics of the study subjects are comprised in Table 1. All test results are comprised in Table 2.

\section{DTC}

The DTC for dual-task conditions that entailed reverse counting (TUGT $\mathrm{TOG}_{\mathrm{COG}}$ ) was $8.4 \%$ higher than the cost of motor-motor tasks (TUGT $\mathrm{TAN}_{\mathrm{MAN}}$ ) in the MCI group, and $30.33 \%$ higher than in the case of the healthy controls unaffected by MCI.

All DTC results are comprised in Table 2.

\section{Single-task performance}

There was a negative, statistically significant correlation between the single-task gait (TUGT) performance and the following factors: both normal and fast gait speed during the 10MWT, strength of the lower limbs (30sChS), static balance with both open and closed eyes,

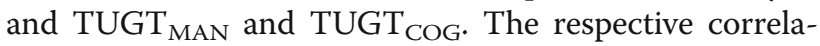
tions were weak (SLS OP, SLS CL), moderate (10MWT - normal speed), strong (10MWT - fast speed, 30sChS), and very strong ( $\mathrm{TUGT}_{\mathrm{MAN}}$ and $\mathrm{TUGT}_{\mathrm{COG}}$ ).

\section{Dual-task performance and functional outcomes in the $\mathrm{MCl}$ group}

Dual-task gait was assessed by means of two tests, i.e. TUGT $_{\text {MAN }}$ and TUGT $T_{\text {COG. }}$. There was a negative, statistically significant correlation between the dual-task motor-motor gait performance (TUGT $\mathrm{TAN}_{\mathrm{MAN}}$ ) and the following factors: both normal and fast gait speed during the 10MWT, the strength of the lower limbs $(30 \mathrm{sChS})$, static balance with both open and closed eyes, and TUGT and TUGT $\mathrm{COG}_{\mathrm{CO}}$. A positive, statistically significant correlation was observed with the FES-I. The respective correlations were weak (FES-I), moderate (10MWT normal and fast speed, 30sChS, SLS OP, SLS CL), strong (30sChS), and very strong (TUGT and TUGT $\mathrm{COG}_{\mathrm{CO}}$ ).

There was a negative, statistically significant correlation between the dual-task motor-cognitive gait performance $\left(\mathrm{TUGT}_{\mathrm{COG}}\right.$ ) and the following factors: both normal and fast gait speed during the 10MWT, the strength of the lower limbs (30sChS), static balance with both open and closed eyes, and TUGT and TUGT ${ }_{\text {MAN }}$. The respective correlations were weak (SLS OP), moderate (10MWT - normal and fast speed, 30sChS, SLS CL), strong (30sChS), and very strong (TUGT and TUGTMAN). Spearman's rank correlation coefficient, functional assessment and Single- and Dual-Task variables are outlined in Table 3. 
Table 1 Baseline characteristics of the study subjects

\begin{tabular}{|c|c|c|c|}
\hline Variable & $\begin{array}{l}\mathrm{MCl} \\
N=44\end{array}$ & $\begin{array}{l}\text { Healthy Group } \\
N=44\end{array}$ & $p$ \\
\hline \multicolumn{4}{|l|}{ Demographic } \\
\hline Gender (female/male\%) & $77 / 23$ & $84 / 16$ & $0.55^{*}$ \\
\hline Age (years),Mean, SD & $83.82(8.5)$ & $81.67(3.5)$ & $0.076^{*}$ \\
\hline Body weight (kg), Mean, SD & $63.90(14.9)$ & $71.83(8)$ & $0.006^{*}$ \\
\hline Body height (cm), Mean, SD & $161.18(9)$ & $161.50(7)$ & $0.971^{*}$ \\
\hline Body mass index $\left(\mathrm{kg} / \mathrm{m}^{2}\right)$ Mean, SD & $24.31(4.8)$ & $27.66(3.6)$ & $0.057^{*}$ \\
\hline \multicolumn{4}{|l|}{ Education } \\
\hline Primary (\%) & 29.5 & 23.8 & $0.091^{* *}$ \\
\hline Secondary (\%) & 18.2 & 38.1 & $0.001^{* *}$ \\
\hline University (\%) & 40.9 & 9.5 & $0.001^{* *}$ \\
\hline Other (vocational training only) (\%) & 11.3 & 14.3 & $0.64^{* *}$ \\
\hline \multicolumn{4}{|l|}{ Marital status } \\
\hline Married (\%) & 15.9 & 14.3 & $0.43^{* *}$ \\
\hline Unmarried (\%) & 11.4 & 4.8 & $0.041^{* *}$ \\
\hline Divorced (\%) & 6.8 & 9.5 & $0.091^{* *}$ \\
\hline Widowed (\%) & 65.9 & 71.4 & $0.14^{* *}$ \\
\hline \multicolumn{4}{|l|}{ Medication } \\
\hline Average $\mathrm{p} /$ day, Mean, SD & $7(5)$ & $5.78(4.7)$ & $0.044^{*}$ \\
\hline$<4$ medication $\mathrm{p} /$ day (\%) & 29.5 & 38.9 & $0.004^{* *}$ \\
\hline$>=4$ medication $\mathrm{p} /$ day (\%) & 70.4 & 61.1 & $0.004^{* *}$ \\
\hline \multicolumn{4}{|l|}{ Concomitant diseases } \\
\hline $1(\%)$ & 47.7 & 47.6 & $0.514^{* *}$ \\
\hline $2(\%)$ & 15.9 & 11.1 & $0.081^{* *}$ \\
\hline$>2(\%)$ & 36.3 & 41.3 & $0.003^{* *}$ \\
\hline \multicolumn{4}{|l|}{ Cognitive status } \\
\hline MMSE (pts.), Mean, SD & $20.34(7.7)$ & $27.72(3.1)$ & $0.001 *$ \\
\hline \multicolumn{4}{|l|}{ Gait Assessment } \\
\hline TUGT (s), Mean, SD & $20.58(8.0)$ & $10.99(4.6)$ & $0.002^{*}$ \\
\hline TUGT $_{\text {MAN }}(\mathrm{s})$, Mean, SD & $24.1(7.9)$ & $11.91(4.5)$ & $0.001^{*}$ \\
\hline TUGT $_{\text {COG }}(\mathrm{s})$, Mean, SD & $25.62(10.4)$ & $14.74(5.5)$ & $0.001 *$ \\
\hline 10MWT normal speed $(\mathrm{m} / \mathrm{s})$, Mean, SD & $0.57(0.2)$ & $0.94(0.3)$ & $0.004^{*}$ \\
\hline 10MWT fast speed $(\mathrm{m} / \mathrm{s})$, Mean, SD & $0.62(0.2)$ & $1.1(0.2)$ & $0.005^{*}$ \\
\hline \multicolumn{4}{|l|}{ FOF } \\
\hline FES-I, Mean, SD & $33.1(15.1)$ & $36.28(3.3)$ & $0.77^{*}$ \\
\hline \multicolumn{4}{|l|}{ Strength of Lower Limbs } \\
\hline 30sChair Stand (n of stand ups), Mean, SD & $9.06(3.8)$ & $9.22(4.5)$ & $0.817^{*}$ \\
\hline \multicolumn{4}{|l|}{ Aerobic Endurance } \\
\hline 2-Min Step (number of steps), Mean, SD & $77.43(42.5)$ & $84.33(44.7)$ & $0.061^{*}$ \\
\hline \multicolumn{4}{|l|}{ Static Balance } \\
\hline SLS OP (s) & $2.52(4.7)$ & $4.04(3.4)$ & $0.119^{*}$ \\
\hline SLS CL (s) & $2.02(4.1)$ & $2.7(3.3)$ & $0.288^{*}$ \\
\hline \multicolumn{4}{|l|}{ Falls } \\
\hline No (\%) & 63.6 & 57.1 & $0.359^{*}$ \\
\hline
\end{tabular}


Table 1 Baseline characteristics of the study subjects (Continued)

\begin{tabular}{|c|c|c|c|}
\hline Variable & $\begin{array}{l}\mathrm{MCl} \\
N=44\end{array}$ & $\begin{array}{l}\text { Healthy Group } \\
N=44\end{array}$ & $p$ \\
\hline $1(\%)$ & 36.4 & 28.6 & $0.359^{*}$ \\
\hline
\end{tabular}

\section{DTC and functional outcomes}

The analysis revealed a moderate, positive and statistically significant correlation between the DTC of motormotor tasks (TUGT $\mathrm{T}_{\text {MAN }}$ ) and single task TUGT.

There was a weak, statistically significant correlation between the DTC of motor-cognitive tasks TUGT.

An adjusted, cross-sectional model was developed to demonstrate the effect of the TUGT alone on the outcome of the dependent variable $\mathrm{DTC}_{\text {MAN }}$ in the subjects affected by MCI.

Around $25 \%$ of the variance of the $\mathrm{DTC}_{\text {MAN }}$ result is accounted for by gait performance in the single-task conditions (TUGT). In the case of Dual Task Cost Cognitive $\left(\mathrm{DTC}_{\mathrm{COG}}\right)$, this value is equal to ca. $10 \%$. A $1 \%$ change in $\mathrm{DTC}_{\mathrm{MAN}}$ corresponds to a ca. $0.5 \mathrm{~s}$ change in TUGT, while a $1 \%$ change in $\mathrm{DTC}_{\mathrm{COG}}$ entails a ca. 0.35 $\mathrm{s}$ change in TUGT walking time. Independent predictors of DTC $_{\text {MAN }}$ are outlined in Table 4.

Table 2 Intergroup differences in the dual-task cost

\begin{tabular}{|c|c|c|c|}
\hline Variable & $\begin{array}{l}\mathrm{MCl} \text { Group } \\
N=44\end{array}$ & $\begin{array}{l}\text { Healthy Group } \\
N=44\end{array}$ & $\mathrm{p}$ \\
\hline \multicolumn{4}{|l|}{ DTC(\%) } \\
\hline DTC $_{\text {MAN }}$, Mean, SD & $17.1(2.7)$ & $8.37(3.1)$ & $0.002^{* *}$ \\
\hline DTC $_{\mathrm{COG}}$, Mean, SD & $25.5(12.1)$ & $38.7(27.6)$ & $0.049 * *$ \\
\hline $\mathrm{DTC}_{\text {MAN }}<15 \%$ & 4.5 & 68 & $0.001^{*}$ \\
\hline $\mathrm{DTC}_{\mathrm{COG}}<15$ & 2.3 & 16.7 & $0.007^{*}$ \\
\hline \multicolumn{4}{|l|}{ Gait speed $(\mathrm{m} / \mathrm{s})$} \\
\hline TUGT, Mean, SD & $0.34(0.2)$ & $0.54(0.5)$ & $0.003^{* *}$ \\
\hline TUGT $_{\text {MAN, Mean, SD }}$ & $0.27(0.2)$ & $0.50(0.4)$ & $0.005^{* *}$ \\
\hline TUGT $C O G$, Mean, SD & $0.26(0.2)$ & $0.41(0.2)$ & $0.003^{* *}$ \\
\hline \multicolumn{4}{|l|}{ Single vs TUG MAN $(\mathrm{m} / \mathrm{s})$} \\
\hline TUGT vs TUGT MAN, Mean, SD & $0.06(0.1)$ & $0.03(0.1)$ & $0.003^{* *}$ \\
\hline $\begin{array}{l}\text { TUGT vs TUGT } \\
\text { Mean, SD }\end{array}$ & $0.07(0.3)$ & $0.13(0.3)$ & $0.416^{* *}$ \\
\hline \multicolumn{4}{|l|}{ Single task vs TUGT $_{\text {MAN }}(\mathrm{n} \%)$} \\
\hline Slower gait speed & 86.4 & 81.6 & $0.641^{*}$ \\
\hline Faster gait speed & 13.6 & 18.4 & $0.511^{*}$ \\
\hline \multicolumn{4}{|l|}{ Single task vs TUGT ${ }_{\mathrm{COG}}(\mathrm{n} \%)$} \\
\hline Slower gait speed & 90.9 & 94.5 & $0.071^{* *}$ \\
\hline Faster gait speed & 9.1 & 5.5 & $0.071^{* *}$ \\
\hline
\end{tabular}

Abbreviations: *Chi2, **U-Mann Whitney
Changes in gait speed between single-task and dual-task conditions

Under both types of dual-task conditions (TUGT MAN $_{\text {N }}$ and TUGT $_{\mathrm{COG}}$ ), there was a weak correlation between both normal and fast gait speed during the 10MWT, the strength of the lower limbs $(30 \mathrm{sChS})$, and gait performance under the single-task conditions.

\section{Discussion}

The results pertaining to functional capacity of the institutionalized older adults affected by MCI correlated with their performance in the dual-task, motor-motor $\left(\mathrm{TUGT}_{\mathrm{MAN}}\right)$, and motor-cognitive (TUGT $\mathrm{T}_{\mathrm{COG}}$ ) activities. The most significant dependences were evident with regard to gait speed (including both normal speed and the fastest speed the subjects were able to attain without putting themselves at a considerable risk of falling), and the strength of the lower limbs assessed by the 30sChS test. The highest decrease in gait speed, i.e. 6.9\%, was found in the subjects examined by the TUGT $\mathrm{TAN}_{\text {MAN }}$ test. Doi et al. investigated the variations in the performance of the dual-task activities and the gait speed observed among the subjects affected by different forms of MCI [22]. Mean decrease in gait speed, while performing an additional task, was $9 \%$.

Doi et al. also demonstrated that gait speed was correlated with working memory, and that visual memory affected both the single-task and the dual-task gait performance, especially in the individuals with amnestic MCI diagnosed against the revised Peterson criteria [23, 24].

The subjects who participated in the present study lost points in the cognitive function test, mostly due to the problems specific to the working and episodic memory, which may give some grounds to believe that these are likely to be the predominant functions related to a reduced gait speed. Aside from memory impairment, overall complexity of the problem was also likely to adversely affect the gait speed, which implies that cognitive load appreciably affected these activities [25].

It is then hardly surprising that - when the TUGT ${ }_{\text {COG }}$ dual-task entailed reverse counting via the subtraction of 7 from a specific number - gait speed decreased by another $6 \%$, as compared to the one during TUGT $_{\text {MAN. In }}$ relation to the single-task test values (TUGT), the respective decreases were 17 and $24 \%$, thereby increasing the corresponding DTC values to $24 \%$ in the case of 
Table 3 Spearman's rank correlation coefficient, functional assessment and Single- and Dual-Task variables in the MCl Group

\begin{tabular}{|c|c|c|c|c|c|c|c|}
\hline & $\begin{array}{l}\text { TUGT } \\
{[s]}\end{array}$ & $\begin{array}{l}\text { TUGT }_{\text {MAN }} \\
{[s]}\end{array}$ & $\begin{array}{l}\text { TUGT }_{\text {COG }} \\
{[\mathrm{s}]}\end{array}$ & $\begin{array}{l}\text { DTC }_{\text {MAN }} \\
{[\%]}\end{array}$ & $\begin{array}{l}\mathrm{DTC}_{\mathrm{COG}} \\
{[\%]}\end{array}$ & $\begin{array}{l}\text { TUGT* }^{*} \text { vs TUGT } \\
\text { [s] }\end{array}$ & $\begin{array}{l}\text { TUGT** vs TUGT } \\
\text { [s] }\end{array}$ \\
\hline MMSE [pts.] & -.12 & -.11 & -.13 & -.09 & -.07 & .12 & .16 \\
\hline $\begin{array}{l}\text { 10MWT normal speed } \\
{[\mathrm{m} / \mathrm{s}]}\end{array}$ & $-.59 * * *$ & $-.57^{* * *}$ & $-.52^{* * *}$ & -.17 & -.12 & $.31^{* * * *}$ & $.32^{* * * *}$ \\
\hline $\begin{array}{l}\text { 10MWT } \\
\text { fast speed } \\
{[\mathrm{m} / \mathrm{s}]}\end{array}$ & $-.60^{* * *}$ & $-.58^{* * *}$ & $-.52^{* * *}$ & -.17 & -.12 & $.32^{* * *}$ & $.33^{* * *}$ \\
\hline $\begin{array}{l}\text { FES-I } \\
\text { [pts.] }\end{array}$ & .26 & .33 & .28 & .07 & .08 & .15 & .20 \\
\hline $\begin{array}{l}\text { 30sChS } \\
\text { [x stand up] }\end{array}$ & $-.62^{* * *}$ & $-.55^{* * *}$ & $-.54^{* * *}$ & -.27 & -.15 & $.38^{* * *}$ & $.35^{* * *}$ \\
\hline $\begin{array}{l}\text { SLS OP* } \\
{[s]}\end{array}$ & $-.37^{* * *}$ & $-.45^{* * *}$ & $-.39 * * *$ & -.00 & -.07 & .12 & .21 \\
\hline $\begin{array}{l}\text { SLS CL* } \\
{[\mathrm{s}]}\end{array}$ & $-.32^{* * *}$ & $-.46^{* * *}$ & $-.40^{* * *}$ & .07 & .03 & .08 & .13 \\
\hline TUGT [s] & 1.00 & $.85^{* * *}$ & $.82^{* * *}$ & $.53^{* * *}$ & .33 & $-.70^{* * *}$ & $-.61^{* * *}$ \\
\hline TUGT $_{\text {MAN }}[\mathrm{s}]$ & $.85^{* * *}$ & 1.00 & $.91^{* * *}$ & .10 & .01 & $-.33^{* * *}$ & $-.32^{* * *}$ \\
\hline $\operatorname{TUGT}_{\mathrm{COG}}[s]$ & $.82^{* * *}$ & $.91^{* * *}$ & 1.00 & .20 & -.15 & $-.39 * * *$ & -.17 \\
\hline
\end{tabular}

Abbreviations: *Differences between gait speed median changes under single-task TUGT and dual-task TUGT Manual conditions, ** Differences between gait speed median changes under single-task TUGT and dual-task TUGT Cognitive conditions ${ }^{* * *} p<0.05$

TUGT $_{\text {MAN }}$ and to $29.44 \%$ in the case of TUGT $\mathrm{TOG}_{\text {COG }}$. The DTC of a secondary task involving reverse counting, as reported by Montero-Odasso et al. for the individuals affected by MCI, was approx. $20 \%$, despite the fact that in this case the subtracted number was 7 , which presumably should have resulted in a higher cost. The $9 \%$ difference between our own result and the one obtained by Montero-Odasso might be attributable to the 7-point difference between the mean MMSE scores (21 and 28 points) [25].

Individuals affected by MCI exhibited a significantly decreased gait speed during the dual-task (DT) motorcognitive activities. Interestingly enough, the DT motormotor activities performed by the MCI subjects while walking seemed to attest to their impaired cognitive abilities. In the present study, $25.7 \%$ of the variance of the TUGT $_{\text {MAN }}$ DTC result was explained by the TUGT (single-task test) result.

Furthermore, Tseng et al. noted that older adults affected by amnestic MCI exhibited a $14 \%$ reduction in gait speed during the dual-task activities; in comparison, the mean reduction in gait speed in a group of older adults characterised by normal cognition was 5\% [26].

Table 4 Independent predictors of DTC MAN

\begin{tabular}{|c|c|c|c|}
\hline \multirow[t]{3}{*}{ Predictor } & \multicolumn{3}{|c|}{$\mathrm{DTC}_{\text {MAN }}(\%)$} \\
\hline & \multicolumn{3}{|c|}{$R^{2}=0.25 P=0.001$} \\
\hline & $\overline{B^{a}}$ & Beta $^{b}$ & P-Value $^{c}$ \\
\hline TUG $[s]$ & 0.54 & 0.53 & 0.001 \\
\hline
\end{tabular}

Abbreviations: $\mathrm{B}^{\mathrm{a}-}$ Unistandardized coefficients, Beta ${ }^{\mathrm{b}}$ - Standard coefficients, $P^{-}$-Value ${ }^{c}$ - Significant value, $R^{2-}$ Coefficient of determination
This gives some grounds to believe that the dual-task tests should actually be applied to assess the gait speed effectively.

It should also be highlighted at this juncture that approx. $10 \%$ of the study participants walked at a faster pace during the motor-cognitive tasks, disregarding it entirely. When conducting investigations pertaining to dual-task activities in the individuals affected by MCI, it should be assumed that $10 \%$ of the subjects disregard the secondary task in favour of completing the primary one, i.e. maintaining balance [5]. This may likely be related to the volume of the prefrontal cortex. Larger cortex volume correlated with higher gait speed during both single-task and dual-task activities, as well as a decreased variance of stride time during the single-task tests.

Besides, the risk of major metabolic disorders in neurotransmitter relations was $63 \%$ in the individuals with significantly decreased gait speed. The likelihood of lower cortex volume in the subjects who achieved average results in the dual-task activities was twice as high. Both the motor cortex and neurotransmitters are involved in the dual-task activities. It should also be noted that when the dual-task activities are performed, the amount of oxyhemoglobin reaching the prefrontal lobe increases, which may be a protective factor in the course of cortical volume loss [26].

There are few studies focused on evaluating functional activity in a broader context. It has clearly been demonstrated that strength of the lower limbs has an appreciable effect on the dual-task performance, as it 
correlates with the difference in the median results of

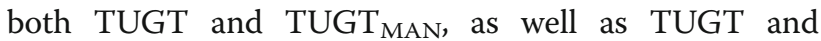
TUGT $_{\text {COG }}$, test pairs. These conclusions are further corroborated by other studies. Casas-Herrero et al. reported a correlation between functional capacity, incidents of falling, muscle mass, muscle strength, and muscle power in the individuals with the frailty syndrome, and concomitant frailty syndrome and MCI [27].

The present Authors established a negative correlation between the strength of the flexor and extensor muscles of the knee $(-0.83, p=0.034$ and $-0.73 p=0.021)$ and the results of the TUGT test, including the version with the reverse counting. Performance in the dual-task activities in TUGT $_{\text {MAN }}$ and TUGT $T_{\text {COG }}$ tests thus depends not only on the memory function and the prefrontal cortex volume, but also on the strength of the lower limbs. Sosnoff expanded the list of relevant factors by age, extent of disability, and gait characteristics, which accounted for $17 \%$ of the variance of DTC [28].

Whereas in the gait characteristics at issue, as yielded by TUGT, this accounted for approx. $25 \%$ of variance in the motor task cost in the model standardised against the select sociodemographic variables.

Finally, it is well-worth highlighting that making (regular) use of the dual-task procedures proves specifically beneficial not only to the individuals affected by MCI, but also to any persons affected by intellectual disability [29].

Further studies on MCI should also take into account the strategies applied by individuals, when increasing and reducing the gait speed during pursuit of dual-task activities. Pertinent reference values for gait speed in the MCI-affected individuals should best be established as a conclusive marker of cognitive dysfunction and impaired functional capacity.

\section{Conclusions}

Functional capacity affected the dual-task performance, especially with regard to the motor-motor tasks.

DTC in the individuals affected by MCI was significantly reduced, being more dependent on the gait speed in the motor-motor tasks, which entail visual memory, than in the motor-cognitive tasks.

\section{Abbreviations \\ MCI: Mild cognitive impairment; ADL: Activities of Daily Living; MCR: Motoric Cognitive Risk; MMSE: Mini-Mental State Examination; TUGT: Timed Up and Go Test; 10MWT: 10 Meter Walk Test; TUGT MAN: Timed Up and Go Manual; TUGT CoG: Timed Up and Go Cognitive; DTC: Dual Task Cost; DTC MAN: Dual

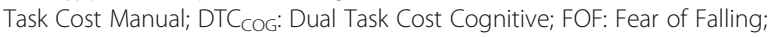 FES-I: Falls Efficacy Scale International Version; 30sChS: 30-s Chair Stand Test; 2-Min Step: 2 Minute Step test; SLS: The Single Leg Stance; SLS OP: The Single Leg Stance Open Eyes; SLS CL: The Single Leg Stance Closed Eyes; DT: dual task}

\section{Acknowledgments}

The Authors are most grateful to all participants for their keen attendance, despite some inconvenience this may well have caused them.

\section{Authors' contributions}

Marek Zak: conceptualization, investigation, methodology, supervision, validation, writing - original draft, writing - review \& editing. Szymon Krupnik: data curation, formal analysis, methodology, project administration, resources, software, visualization, writing - original draft. Waldemar Brola: investigation, resources, validation. Dorota Rebak: data curation, formal analysis, Tomasz Sikorski: software, visualization. Frederic Dutheil: resources, writing - original draft, Jarosław Andrychowski: project administration, resources, software, Daniel Courteix: conceptualization, methodology, writing - original draft. All Authors reviewed and approved the final version of the manuscript, and agreed to be held accountable for all aspects of the work.

\section{Funding}

The project is supported under the programme established by the Minister of Science and Higher Education, named "Regional Initiative of Excellence", spanning the period 2019-2022; Project No 024/RID/2018/19; amount of financing: PLN 11999 000.00.

\section{Availability of data and materials}

The datasets generated and/or analysed during the current study are not publicly available due to institutional restrictions, although they might be made available by the Corresponding Author upon a reasonable request.

\section{Declarations}

Ethics approval and consent to participate

The local Bioethics Review Committee, having assessed the study design, judged that ethics approval was not required under presently applicable legislation, nevertheless it endorsed in principle the study protocol (Decision Ref. No. 40/KBL/OIL/2017). All participants were furnished with detailed information on the aims and methods to be used throughout the study, and gave their written informed consent to participate.

\section{Consent for publication}

The Authors represent that Consent for publication statement is not justified by the study protocol, as all source data are provided in an anonymised format.

With the kindest regards,

Marek ZAK.

(Corresponding Author)

Marek ZAK, MsPT, PhD, Ass. Professor,

The Institute of Health Sciences, Collegium Medicum.

The Jan Kochanowski University,

ul. Zeromskiego 5, 25-369 Kielce, Poland,

E-mail: mzak1@onet.eu

Phone: (+ 48) 413496909 Fax: (+48) 413496916.

\section{Competing interests}

The Authors report no conflicts of interest in this work.

\section{Author details}

${ }^{1}$ The Institute of Health Sciences, Collegium Medicum, The Jan Kochanowski University, ul. Zeromskiego 5, 25-369 Kielce, Poland. Symmetry, Medical Rehabilitation Centre, Sosnowiec, Poland. ${ }^{3}$ CNRS, LaPSCo, Physiological and Psychosocial Stress, Université Clermont Auvergne, Clermont- Ferrand, France. ${ }^{4}$ Departement de Médecine du travail, Clermont-Ferrand University Hospital, G. Montpied Hospital, Clermont-Ferrand, France. ${ }^{5}$ Laboratory of the Metabolic Adaptations to Exercise under Physiological and Pathological Conditions (AME2P - EA 3533), University Clermont Auvergne,

Clermont-Ferrand, France.

Received: 2 March 2021 Accepted: 29 June 2021

Published online: 12 July 2021

\section{References}

1. Cedervall Y, Stenberg AM, Ahman HB, Giedraitis V, Tinmark F, Berglund L, et al. Timed up-and-go dual-task testing in the assessment of cognitive function: a mixed methods observational study for development of the UDDGait protocol. Int J Environ Res Public Health. 2020;17(5):1715. https:// doi.org/10.3390/ijerph17051715. 
2. Winblad B, Palmer K, Kivipelto M, Jelic V, Fratiglioni L, Wahlund LO, et al. Mild cognitive impairment--beyond controversies, towards a consensus: report of the international working group on mild cognitive impairment. J Intern Med. 2004;256(3):240-6. https://doi.org/10.1111/j.1365-2796.2004.013 80.x.

3. Langa KM, Levine DA. The diagnosis and management of mild cognitive impairment: a clinical review. JAMA. 2014;312(23):2551-61. https://doi.org/1 0.1001/jama.2014.13806.

4. Cahill A, Diaz-Ponce AM, Coen RF, Walsh C. The underdetection of cognitive impairment in nursing homes in the Dublin area. The need for on-going cognitive assessment. Age Ageing. 2010;39(1):128-31. https://doi.org/10.1 093/ageing/afp198.

5. Srygley JM, Mirelman A, Herman T, Giladi N, Hausdorff JM. When does walking alter thinking? Age and task associated findings. Brain Res. 2009; 9(1253):92-9. https://doi.org/10.1016/j.brainres.2008.11.067.

6. Montero-Odasso M, Bergman H, Phillips NA, Wong CH, Sourial N, Chertkow $\mathrm{H}$. Dual-tasking and gait in people with mild cognitive impairment. The effect of working memory. BMC Geriatrics. 2009;1 (9):41. https://doi.org/10.11 86/1471-2318-9-41.

7. Lee J, Park S. Effects of a priority-based dual task on gait velocity and variability in older adults with mild cognitive impairment. J Exerc Rehabil. 2018;14(6):993-7. https://doi.org/10.12965/jer.1836402.201.

8. Shim H, Kim M, Won C. Motoric cognitive risk syndrome is associated with processing speed and executive function, but not delayed free recall memory: the Korean frailty and aging cohort study (KFACS). Arch Gerontol Geriatr. 2020;87:103990. https://doi.org/10.1016/j.archger.2019.103990.

9. Chhetri JK, Chan P, Vellas B, Cesari M. Motoric Cognitive Risk Syndrome: Predictor of Dementia and Age-Related Negative Outcomes. Front Med (Lausanne). 2017;4:166. https://doi.org/10.3389/fmed.2017.00166.

10. Crum RM, Anthony JC, Bassett SS, Folstein MF, Anthony, Bassett, et al. Population-based norms for the mini-mental status examination by age and educational level. JAMA. 1993;269(18):2386-91. https://doi.org/10.1001/ja ma.1993.03500180078038.

11. Folstein MF, Folstein SE, McHugh PR. "Mini-mental state".A practical method for grading the cognitive state of patients for the clinician. J Psychiatr Res. 1975;12(3):189-98. https://doi.org/10.1016/0022-3956(75)90026-6.

12. Podsiadlo $D$, Richardson $S$. The timed "up \& go": a test of basic functional mobility for frail elderly persons. J Am Geriatr Soc. 1991;39(2):142-8. https:// doi.org/10.1111/j.1532-5415.1991.tb01616.x.

13. Shumway-Cook A, Brauer S, Woolacott M. Predicting the Probability for Falls in Community - Dwelling Older Adults Using the Timed Up \& Go Test. Physical Therapy. 2002;80:896-903. https://doi.org/10.1093/ptj/80.9.896

14. Bock O. Dual-task costs while walking increase in old age for some, but not for other tasks: an experimental study of healthy young and elderly persons. J NeuroEng Rehabil. 2008;13(5):27. https://doi.org/10.1186/1743-0003-5-27.

15. Hofheinz $M$, Schusterschitz $C$. Dual task interference in estimating the risk of falls and measuring change: a comparative, psychometric study of four measurements. ClinRehabil. 2010;24(9):831-42. https://doi.org/10.1177/02 69215510367993.

16. Watson MJ. Refining the ten-metre walking test for use with neurologically impaired people. Physiotherapy. 2002;88,7:386-397. https://doi.org/10.1016/ S0031-9406(05)61264-3

17. Yardley L, Beyer N, Hauer K, Kempen G, Piot-Ziegler C, Todd C. Development and initial validation of the falls efficacy scale-international (FES-I). Age Ageing. 2005;34(6):614-9. https://doi.org/10.1093/ageing/afi196.

18. Zak M. Fear of falling among the elderly: evaluation of the assessment methods. Med Rehabil. 2014;18(2):17-22 ISSN 1427-9622.

19. Jones CJ, Rikli RE. The application of Fullerton's Functional Fitness Test for older adults in a group setting. Science \& Sports. 2000;15:194-197. https:// doi.org/10.1016/S0765-1597(00)80005-2

20. Rikli RE, Jones CJ. Development and validation of a functional fitness test for community-residing older adults. J Aging Phys Act. 1999;7(2):129-61. https://doi.org/10.1123/japa.7.2.129.

21. Jacobs V, Horak FB, Tran VK, Nutt JG. Multiple balance tests improve the assessment of postural stability in subjects with Parkinson's disease. J Neurol Neurosurg Psychiatry. 2006;77(3):322-6. https://doi.org/10.1136/jnnp.2005. 068742.

22. Doi T, Shimada H, Makizako H, Tsutsumimoto K, Hotta R, Nakakubo S, et. al. Effects of white matter lesions on trunk stability during dual-task walking among older adults with mild cognitive impairment. Age (Dordr). 2015;37,6: 120. doi: https://doi.org/10.1007/s11357-015-9858-x.
23. Petersen RC. Mild cognitive impairment as a diagnostic entity. Journal of Internal Medicine. 2004;256,3:183-94. https://doi.org/10.1111/j.1365-2796.2 004.01388.x

24. Montero-Odasso M, Casas A, Hansen KT, Bilski P, Gutmanis I, Wells JL, et. al. Quantitative gait analysis under dual-task in older people with mild cognitive impairment: a reliability study. Journal of NeuroEngineering and Rehabilitation 2009;6:35. doi: https://doi.org/10.1186/1743-0003-6-35, 1.

25. Tseng BY, Cullum CM, Zhang R. Older adults with amnestic mild cognitive impairment exhibit exacerbated gait slowing under dual-task challenges. Curr Alzheimer Res. 2014;11(5):494-500. https://doi.org/10.2174/1567205011 666140505110828.

26. Doi T, Makizako H, Shimada H, Park H, Tsutsumimoto K, Uemura K, et. al. Brain activation during dual-task walking and executive function among older adults with mild cognitive impairment: afNIRS study. Aging Clin Exp Res 2013;25,5:539-544. doi: https://doi.org/10.1007/s40520-013-0119-5.

27. Casas-Herrero A, Cadore EL, Zambom-Ferraresi F. Functional capacity, muscle fat infiltration, power output and cognitive impairment in institutionalized frail oldest-old. Rejuvenation Res. 2013;16(5):396-403. https://doi.org/10.1089/rej.2013.1438.

28. Sosnoff JJ, Socie MJ, Sandroff BM, Balantrapu S, Suh Y, Pula JH, et. al. Mobility and cognitive correlates of dual task cost of walking in persons with multiple sclerosis. Disabil Rehabil 2014;36,3:205-209. doi: https://doi. org/10.3109/09638288.2013.782361.

29. Mikołajczyk E, Jankowicz-Szymanska A. Dual-task functional exercises as an effective way to improve dynamic balance in persons with intellectual disability - continuation of the project. Medical Studies/StudiaMedyczne. 2017;33(2):102-9. https://doi.org/10.5114/ms.2017.68703.

\section{Publisher's Note}

Springer Nature remains neutral with regard to jurisdictional claims in published maps and institutional affiliations.
Ready to submit your research? Choose BMC and benefit from:

- fast, convenient online submission

- thorough peer review by experienced researchers in your field

- rapid publication on acceptance

- support for research data, including large and complex data types

- gold Open Access which fosters wider collaboration and increased citations

- maximum visibility for your research: over $100 \mathrm{M}$ website views per year

At BMC, research is always in progress.

Learn more biomedcentral.com/submissions 